\title{
PERIODIC COMPLEXES AND GROUP ACTIONS
}

\author{
ALEJANDRO ADEM AND JEFF H. SMITH
}

\begin{abstract}
In this paper we show that the cohomology of a connected $C W$-complex is periodic if and only if it is the base space of a spherical fibration with total space that is homotopically finite dimensional. As applications we characterize those discrete groups that act freely and properly on $\mathbb{R}^{n} \times \mathbb{S}^{m}$; we construct non-standard free actions of rank two simple groups on finite complexes $Y \simeq \mathbb{S}^{n} \times \mathbb{S}^{m}$; and we prove that a finite $p$-group $P$ acts freely on such a complex if and only if it does not contain a subgroup isomorphic to $(\mathbb{Z} / p)^{3}$.
\end{abstract}

\section{INTRODUCTION}

Let $G$ be a finite group for which all abelian subgroups are cyclic. Swan [30] has proved that any such group acts freely on a finite complex $X \simeq \mathbb{S}^{m}$ for some $m>0$. These groups are best described as those having periodic group cohomology. Since then there have been numerous attempts to extend this type of result to other classes of groups, including (1) infinite groups with periodic cohomology and (2) finite groups with non-periodic cohomology.

In this paper we prove a homotopy-theoretic characterization of cohomological periodicity, a notion which we now make precise.

Definition 1.1. A CW-complex $X$ has periodic cohomology if there is a cohomology class $\alpha \in H^{*}(X, \mathbb{Z})$ with $|\alpha|>0$ and an integer $d \geq 0$ such that the map

$$
\alpha \cup-: H_{l o c}^{n}(X, \mathcal{B}) \rightarrow H_{l o c}^{n+|\alpha|}(X, \mathcal{B})
$$

induced by the cup product with $\alpha$ is an isomorphism for every local coefficient system $\mathcal{B}$ and every integer $n \geq d$.

Our main result is

Theorem 1.2. Let $X$ be a connected $C W$-complex. The cohomology of $X$ is periodic if and only if there is a spherical fibration $E \rightarrow X$ with a total space $E$ that is homotopy equivalent to a finite dimensional $C W$-complex.

In particular if $\Gamma$ is a discrete group, then a $C W$-complex of type $K(\Gamma, 1)$ has periodic cohomology if and only if $\Gamma$ acts freely and properly on a finite dimensional complex (i.e. a free $\Gamma-\mathrm{CW}$ complex) that is homotopy equivalent to a sphere. From this we obtain a characterization of groups which act freely and properly on $\mathbb{R}^{n} \times \mathbb{S}^{m}$ which extends a conjecture formulated by Wall [33] for groups of finite virtual cohomological dimension:

Both authors were partially supported by grants from the NSF and the CRM-Barcelona. 
Corollary 1.3. A discrete group $\Gamma$ acts freely and properly on $\mathbb{R}^{n} \times \mathbb{S}^{m}$ for some $m, n>0$ if and only if $\Gamma$ is a countable group with periodic cohomology.

Our results apply to natural situations arising from finite group actions. We consider actions that have isotropy subgroups with periodic cohomology.

Theorem 1.4. Let $G$ be a finite group and let $X$ be a finite dimensional $G-C W$ complex such that every abelian subgroup of an isotropy subgroup is cyclic. Then for some integer $N>0$ there is a finite dimensional $C W$-complex $Y$ such that $Y \simeq \mathbb{S}^{N} \times X$ and a free action of $G$ on $Y$ such that the projection $Y \rightarrow X$ is $G$-equivariant. If $X$ is simply-connected and finitely dominated then $Y$ can be taken to be a finite complex.

The rank $r(G)$ of a finite group $G$ is the dimension of its largest elementary abelian subgroup. It has been conjectured (see [5]) that Swan's theorem admits the following generalization: if $k$ is the smallest integer such that a finite group $G$ will act freely on a finite complex $Y \simeq$ $\mathbb{S}^{n_{1}} \times \cdots \times \mathbb{S}^{n_{k}}$, then $k=r(G)$. Using linear spheres, we prove:

Theorem 1.5. Let $G$ denote a finite $p$-group with center $Z(G)$ such that $r(G)-r(Z(G)) \leq 1$; then $G$ acts freely on a finite complex $Y \simeq \mathbb{S}^{N} \times\left(\mathbb{S}^{2[G: Z(G)]-1}\right)^{r(G)-1}$.

As a corollary we obtain a succinct geometric characterization of low-rank $p$-groups which represents a long-sought extension of Swan's theorem beyond spherical space forms:

Corollary 1.6. Let $G$ denote a finite p-group. Then $G$ acts freely on a finite complex $Y \simeq$ $\mathbb{S}^{n} \times \mathbb{S}^{m}$ if and only if $G$ does not contain a subgroup isomorphic to $\mathbb{Z} / p \times \mathbb{Z} / p \times \mathbb{Z} / p$.

Constructing free actions of simple groups is a particularly difficult problem. Using the classification of finite simple groups [17] and a result due to Oliver [23] we show there is no free action of a rank two simple group on a product of spheres $\mathbb{S}^{n} \times \mathbb{S}^{m}$ that is a product action. Using character theory, we construct free actions of some simple groups which are therefore necessarily exotic (i.e. non-product actions).

Theorem 1.7. Each of the simple groups $A_{5}, S L_{3}\left(\mathbb{F}_{2}\right), U_{3}(3)$, and $U_{3}(4)$ acts on linear spheres with rank one isotropy. Hence each acts freely and exotically on a finite complex that is homotopy equivalent to a product of spheres $\mathbb{S}^{n} \times \mathbb{S}^{m}$.

Unfortunately many of the rank two simple groups do not have linear spheres with the necessary properties. To deal with such cases, we develop a method to produce $p$-local Euler classes from local subgroups. We then assemble the actions on $p$-local spheres to produce an action on a homotopy sphere with an appropriate integral Euler class. Concentrating on the prime 2 (indeed every non-abelian simple group contains a copy of $\mathbb{Z} / 2 \times \mathbb{Z} / 2$ ) we use the poset of elementary abelian subgroups to produce suitable Euler classes 2-locally. Using additional arguments for odd primes and making a case-by-case analysis we obtain

Theorem 1.8. Let $G$ denote a rank two simple group other than $P S L_{3}\left(\mathbb{F}_{p}\right), p$ an odd prime. Then $G$ acts freely on a finite complex $Y \simeq \mathbb{S}^{n} \times \mathbb{S}^{m}$. 
Our methods also imply

Theorem 1.9. Let $G$ denote a rank two finite group such that all of its Sylow subgroups are either normal in $G$, abelian or generalized quaternion. Then $G$ acts freely on a finite complex $Y \simeq \mathbb{S}^{n} \times \mathbb{S}^{m}$

We are grateful to the CRM-Barcelona for its hospitality and to W.Dwyer, G.Mislin, B.Oliver, W.Lück, R.Solomon, G.Seitz, S.Smith and O.Talelli for their helpful comments. We also thank the referee for his useful remarks. The results in this paper have been announced in [3].

\section{PERIODIC COMPLEXES}

In this section we generalize a result of Swan [30] concerning finite groups with periodic cohomology. When coefficients do not appear in a (co)homology group we assume that they are in $\mathbb{Z}$, without any twisting. We first prove a number of lemmas required for our main results.

Definition 2.1. A CW-complex $X$ has periodic cohomology if there is a cohomology class $\alpha \in H^{*}(X)$ with $|\alpha|>0$ and an integer $d \geq 0$ such that the map

$$
\alpha \cup-: H_{l o c}^{n}(X, \mathcal{B}) \rightarrow H_{l o c}^{n+|\alpha|}(X, \mathcal{B})
$$

induced by the cup product with $\alpha$ is an isomorphism for every local coefficient system $\mathcal{B}$ and every integer $n \geq d$.

A spherical fibration is a Serre fibration $E \rightarrow B$ for which the fiber is homotopy equivalent to a sphere $\mathbb{S}^{m}$. A spherical fibration is orientable if the action of $\pi_{1}(B)$ on $H^{m}\left(\mathbb{S}^{m}\right)$ is trivial and an orientation of the spherical fibration is a choice of generator for $H^{m}\left(\mathbb{S}^{m}\right)$. The Euler class of an oriented spherical fibration is the cohomology class in $H^{m+1}(B)$ that is the transgression of the generator for $H^{m}\left(\mathbb{S}^{m}\right)$.

We recall some basic constructions in homotopy theory. Note that we will be working in the homotopy category throughout, so diagrams and constructions which we use should be understood accordingly. We recall the notion of the fiber join of two fibrations.

Definition 2.2. Let $E_{1} \rightarrow X$ and $E_{2} \rightarrow X$ be two fibrations over the same base space $X$, and let $E_{1} \times_{X} E_{2}$ be their fibered product. The fiber join of these fibrations, denoted by $E_{1} *_{X} E_{2} \rightarrow X$, is defined as the homotopy pushout of the diagram of fibrations over $X$

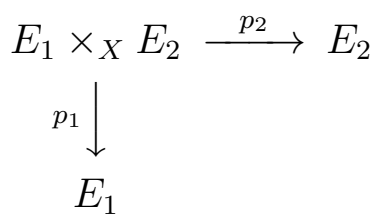

where $p_{1}$ and $p_{2}$ are the natural projections. The fibration $E_{1} *_{X} E_{2} \rightarrow X$ has fiber $F_{1} * F_{2}$, the join of the respective fibers of the two original fibrations. 
In what follows we will make use of basic properties of Postnikov sections (see [18] for background). The $q$-th Postnikov section of the $m$-sphere is denoted $P_{q} \mathbb{S}^{m}$. Recall that the induced map $H^{m}\left(P_{q} \mathbb{S}^{m}\right) \rightarrow H^{m}\left(\mathbb{S}^{m}\right)$ is an isomorphism if $q \geq m$.

Definition 2.3. Let $X$ be a connected CW-complex and let $k \geq 0$ be an integer. A cohomology class $\alpha \in H^{n}(X)$ is a $k$-partial Euler class if there is a fibration sequence

$$
P_{k+n-1} \mathbb{S}^{n-1} \rightarrow E \rightarrow X
$$

such that the action of $\pi_{1}(X)$ on $H^{n-1}\left(P_{k+n-1} \mathbb{S}^{n-1}\right)=\mathbb{Z}$ is trivial and a generator of this group transgresses to $\alpha$.

Proposition 2.4. For an integer $q \geq m$, let $P_{q} \mathbb{S}^{m} \rightarrow E \rightarrow B$ be a fibration sequence with a connected base. If the action of $\pi_{1}(B)$ on $H^{m}\left(P_{q} \mathbb{S}^{m}\right)$ is trivial then the action of $\pi_{1}(B)$ on all the groups $H^{*}\left(P_{q} \mathbb{S}^{m}\right)$ is trivial.

Proof. For any fibration sequence $F \rightarrow E \rightarrow B$ there is a natural map $\pi_{1}(B) \rightarrow[F, F]$ and the action of $\pi_{1}(B)$ on $H^{*}(F)$ is determined by the composition $\pi_{1}(B) \rightarrow[F, F] \rightarrow$ $\operatorname{Hom}\left(H^{*}(F), H^{*}(F)\right)$. The functor $P_{m}$ induces a map $[F, F] \rightarrow\left[P_{m} F, P_{m} F\right]$; in the proposition, $F=P_{q} \mathbb{S}^{m}, P_{m} F=K(\mathbb{Z}, m)$ and $\left[P_{m} F, P_{m} F\right]=\mathbb{Z}$. So the functor $P_{m}$ induces an isomorphism $[F, F] \rightarrow \mathbb{Z}$ (indeed, for $q \geq m$, we have isomorphisms $\left[\mathbb{S}^{m}, \mathbb{S}^{m}\right] \cong\left[P_{q} \mathbb{S}^{m}, P_{q} \mathbb{S}^{m}\right]$ by the fundamental property of Postnikov sections). The action of $\pi_{1}(B)$ on $H^{m}\left(P_{q} \mathbb{S}^{m}\right)$ determines the homomorphism $\pi_{1}(B) \rightarrow[F, F]=\mathbb{Z}$; if the action is trivial then the homomorphism is trivial and the action of $\pi_{1}(B)$ on $H^{*}\left(P_{q} \mathbb{S}^{m}\right)$ is trivial.

Lemma 2.5. Let $X$ be a connected $C W$-complex and let $k \geq 0$ be an integer. For every cohomology class $\alpha \in H^{*}(X)$ of positive degree there is an integer $q \geq 1$ such that the cup power $\alpha^{q}$ is a $k$-partial Euler class.

Proof. The lemma is proved by induction on $k$. For $k=0$, let $f: X \rightarrow K(\mathbb{Z}, n)$ be a map that represents the cohomology class $\alpha$ and let $E \rightarrow X$ be the fibration defined by the pullback square

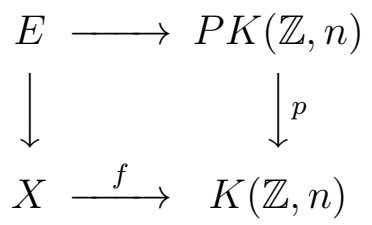

where $p$ is the path fibration. The fiber of $E \rightarrow X$ is $K(\mathbb{Z}, n-1)$ which is $P_{n-1} \mathbb{S}^{n-1}$. By construction the generator of $H^{n-1}(K(\mathbb{Z}, n-1))$ transgresses to $\alpha \in H^{*}(X)$. Hence every $\alpha$ is a $0-$ partial Euler class.

The inductive step is next. Assuming that $\alpha \in H^{n}(X)$ is a $k$-partial Euler class, we show that some cup power $\alpha^{q}$ is a $k+1$-partial Euler class. Let

$$
P_{k+n-1} \mathbb{S}^{n-1} \rightarrow E \rightarrow X
$$

be a fibration sequence for which a generator of $H^{n-1}\left(P_{k+n-1} \mathbb{S}^{n-1}\right)$ transgresses to $\alpha$. By Proposition 2.4, the action of $\pi_{1}(X)$ on $H^{*}\left(P_{k+n-1} \mathbb{S}^{n-1}\right)$ is trivial. So, the $E_{2}$ term of the Serre spectral 
sequence for cohomology with coefficients in $\pi_{k+n}\left(\mathbb{S}^{n-1}\right)$ is

$$
E_{2}^{p, q}=H^{p}\left(X, H^{q}\left(P_{k+n-1} \mathbb{S}^{n-1}, \pi_{n+k}\left(\mathbb{S}^{n-1}\right)\right)\right) .
$$

Let $g_{k}: P_{k+n-1} \mathbb{S}^{n-1} \rightarrow K\left(\pi_{k+n}\left(\mathbb{S}^{n-1}\right), k+n+1\right)$ be the $k$-invariant and let $\gamma_{k}$ denote the cohomology class represented by $g_{k}$. The class $\gamma_{k}$ lies in $E_{2}^{0, k+n+1}$ and survives to $E_{k+3}$. The differential $d_{k+3} \gamma_{k}$ is a class in $E_{k+3}^{k+3, n-1}$; if $k$ is sufficiently large, this group is isomorphic to $H^{k+3}\left(X, \pi_{n+k}\left(\mathbb{S}^{n-1}\right)\right)$ and $\alpha \cup d_{k+3} \gamma_{k}=0$. If $d_{k+3} \gamma_{k}=0$ then $\gamma_{k}$ is transgressive and $d_{k+n+2} \gamma_{k} \in H^{*}(X) /(\alpha)$.

Consider the diagram

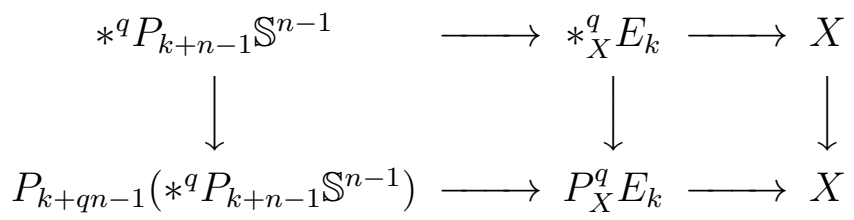

having as its first row the $q$-fold fiberwise join of the fibration $E_{k} \rightarrow X$ and as its second row the fibration obtained by the fiberwise application of the Postnikov section functor $P_{k+q n-1}$ to the first row. Applying the Postnikov section functor to the natural map $\mathbb{S}^{q n-1} \rightarrow *^{q} P_{k+n-1} \mathbb{S}^{n-1}$ gives a weak equivalence

$$
P_{k+q n-1} \mathbb{S}^{q n-1} \rightarrow P_{k+q n-1}\left(*^{q} P_{k+n-1} \mathbb{S}^{n-1}\right) .
$$

In dimension $k+q n+1$ one has

$$
\begin{aligned}
H_{k+q n+1}\left(*^{q} P_{k+n-1} \mathbb{S}^{n-1}\right) & =\oplus_{q} \pi_{k+n}\left(\mathbb{S}^{n-1}\right) \\
H_{k+q n+1}\left(P_{k+q n-1} \mathbb{S}^{q n-1}\right) & =\pi_{k+q n}\left(\mathbb{S}^{q n-1}\right) .
\end{aligned}
$$

The induced map in homology is the $q$-fold sum of maps $f_{i}: \pi_{k+n}\left(\mathbb{S}^{n-1}\right) \rightarrow \pi_{k+q n}\left(\mathbb{S}^{q n-1}\right)$. By symmetry $f_{i}=f_{j}$ for all $1 \leq i, j \leq q$. To understand $f_{1}$ consider the composite

$$
P_{k+n-1} \mathbb{S}^{n-1} *\left(*^{q-1} \mathbb{S}^{n-1}\right) \rightarrow *^{q} P_{k+n-1} \mathbb{S}^{n-1} \rightarrow P_{k+q n-1} \mathbb{S}^{q n-1} .
$$

The adjoint is the map

$$
P_{k+n-1} \mathbb{S}^{n-1} \rightarrow \Omega^{(q-1) n} P_{k+q n-1} \mathbb{S}^{q n-1}=P_{k+n-1} \Omega^{(q-1) n} \mathbb{S}^{q n-1}
$$

which is determined on the bottom cell. It is obtained by applying the functor $P_{k+n-1}$ to the inclusion $\mathbb{S}^{n-1} \rightarrow \Omega^{(q-1) n} \mathbb{S}^{q n-1}$. It follows that $f_{1}$ is the suspension homomorphism and therefore that the map $\oplus f_{i}$ is the fold map followed by the suspension homomorphism.

Let $g_{k}^{[q]}$ denote the $k$-invariant

$$
P_{k+q n-1} \mathbb{S}^{q n-1} \rightarrow K\left(\pi_{k+q n}\left(\mathbb{S}^{q n-1}\right), k+q n+1\right),
$$

and let $\gamma_{k}^{[q]}$ be the cohomology class that it represents. By naturality of differentials it follows that $d_{k+3} \gamma_{k}^{[q]}$ is the image of $q d_{k+3} \gamma_{k}$ under the map

$$
H^{k+3}\left(X, \pi_{k+n}\left(\mathbb{S}^{n-1}\right)\right) \rightarrow H^{k+3}\left(X, \pi_{k+q n}\left(\mathbb{S}^{q n-1}\right)\right)
$$

that is induced by the suspension homomorphism on the coefficients. 
Now the image of the suspension homomorphism is a finite group and if $q$ is the exponent of the image then $d_{k+3} \gamma_{k}^{[q]}=0$. On the other hand, if $d_{k+3} \gamma_{k}=0$ then $\gamma_{k}$ transgresses to a class $\delta \in H^{k+n+2}(X)$ which is well defined modulo the ideal generated by $\alpha$ and $\gamma_{k}^{[q]}$ transgresses to $q \alpha^{q-1} \bar{\delta}$ modulo the ideal generated by $\alpha^{q}$ where $\bar{\delta}$ is the image of $\delta$ under the map

$$
H^{k+n+2}\left(X, \pi_{k+n}\left(\mathbb{S}^{n-1}\right)\right) \rightarrow H^{k+n+2}\left(X, \pi_{k+q n}\left(\mathbb{S}^{q n-1}\right)\right)
$$

induced by the suspension homomorphism. Again, if $q$ is the exponent of the image of the suspension homomorphism then $\gamma_{k}^{[q]}$ transgresses to zero modulo the ideal generated by $\alpha^{q}$.

Let us now choose $q$ to be the square of the exponent of the image of the suspension homomorphism. Then by construction, $\gamma_{k}^{[q]}$ survives to $E_{\infty}$ in the Serre spectral sequence. This implies that the map $g_{k}^{[q]}$ extends to a map on $P_{X}^{q} E_{k}$ and there is a diagram

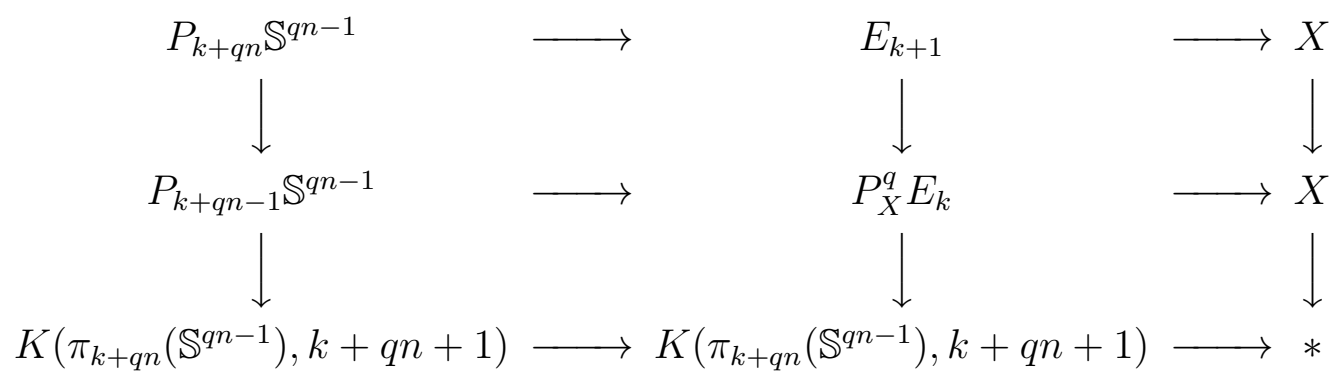

in which each row and each column is a fibration sequence. Taking the fibration sequence in the first row completes the inductive step.

Lemma 2.6. Let $P_{k+|\alpha|-1} \mathbb{S}^{|\alpha|-1} \rightarrow E \rightarrow X$ be a fibration sequence such that a generator of $H^{|\alpha|-1}\left(P_{k+|\alpha|-1} \mathbb{S}^{|\alpha|-1}\right)$ transgresses to $\alpha \in H^{*}(X)$. If $k \geq d-2$ and the map $\alpha \cup-: H^{m}(X) \rightarrow$ $H^{m+|\alpha|}(X)$ is an isomorphism in integral cohomology for $m \geq d$ then there is an orientable spherical fibration $\bar{E} \rightarrow X$ with Euler class $\alpha$. If in addition the map $\alpha \cup-: H_{\text {loc }}^{m}(X, \mathcal{B}) \rightarrow$ $H_{\text {loc }}^{m+|\alpha|}(X, \mathcal{B})$ is an isomorphism for every local coefficient system $\mathcal{B}$ and every integer $m \geq d$ then $\bar{E}$ is homotopy equivalent to a $C W$-complex of dimension less than $d+|\alpha|$.

Proof. Let $n=|\alpha|$; then for each integer $t \geq k+n-1$ we will construct by induction a fibration sequence $P_{t} \mathbb{S}^{n-1} \rightarrow E_{t} \rightarrow X$ and a map $E_{t+1} \rightarrow E_{t}$ over $X$. The induction begins at $t=k+n-1$ with the fibration sequence $P_{k+n-1} \mathbb{S}^{n-1} \rightarrow E \rightarrow X$ that one has by hypothesis.

The inductive step is next. Suppose that one has a fibration sequence $P_{t} \mathbb{S}^{n-1} \rightarrow E_{t} \rightarrow X$. The $k$-invariant $g_{t}: P_{t} \mathbb{S}^{n-1} \rightarrow K\left(\pi_{t+1}\left(\mathbb{S}^{n-1}\right), t+2\right)$ extends to a map on $E_{t}$ if and only if the cohomology class represented by $g_{t}$ survives to $E_{\infty}$ in the Serre spectral sequence for cohomology with coefficients in $\pi_{t+1}\left(\mathbb{S}^{n-1}\right)$. There are two differentials which might be non-trivial on the class represented by $g_{t}$. These are $d_{t-n+4}$, taking values in the kernel of multiplication by $\alpha$; and $d_{t+3}$, taking values in $H^{*}(X) /(\alpha)$. But by hypothesis both obstruction groups are zero and one can 
construct the diagram

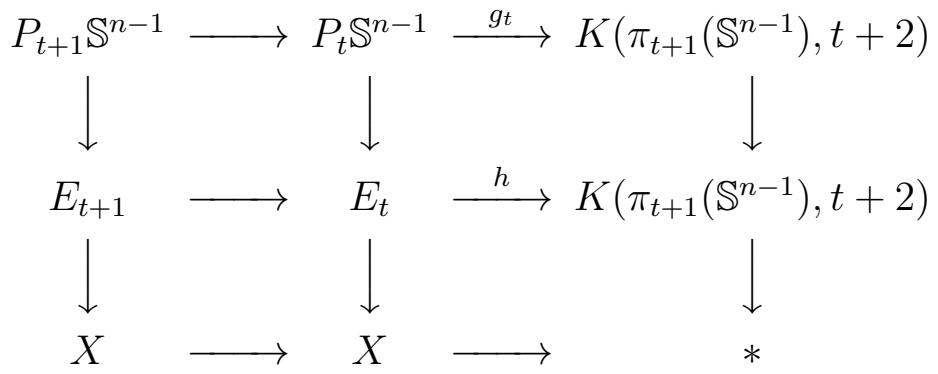

in which each row and each column is a fibration sequence.

Let $E_{\infty}=\operatorname{holim}_{t} E_{t}$. The homotopy fiber of the map $E_{\infty} \rightarrow X$ is weakly equivalent to $\mathbb{S}^{n-1}$. By construction the integral cohomology of $E_{\infty}$ vanishes above dimension $d+|\alpha|-1$. If in addition one has the condition on cohomology with local coefficients that is given in the hypotheses, then it follows from the Serre spectral sequence with local coefficients that $E_{\infty}$ has finite cohomological dimension. So by a result of Wall [32], $E_{\infty}$ is homotopy equivalent to a finite dimensional $\mathrm{CW}$ complex. From this we obtain a spherical fibration over $X$ with total space $\bar{E}$ homotopy equivalent to a complex of dimension less than $d+|\alpha|$.

Lemma 2.7. Let $X$ be a $C W$-complex and let $E \rightarrow X$ be an orientable fibration such that the fiber is equivalent to an odd dimensional sphere other than $\mathbb{S}^{1}$, so $\pi_{1}(E)=\pi_{1}(X)$. For each integer $q \geq 1$, the $q$-fold fiber join $*_{X}^{q} E \rightarrow X$ is a spherical fibration. If $E$ is finitely dominated then $*{ }_{X}^{q} E$ is finitely dominated and if $\mathcal{O}_{E} \in \widetilde{K}_{0}\left(\mathbb{Z} \pi_{1}(X)\right)$ is the finiteness obstruction of $E$ then $q \mathcal{O}_{E}$ is the finiteness obstruction of $*_{X}^{q} E$.

Proof. Let $E_{1} \rightarrow X$ and $E_{2} \rightarrow X$ be orientable spherical fibrations with fibers that are odd dimensional spheres of dimension greater than one. Then there is a pushout square

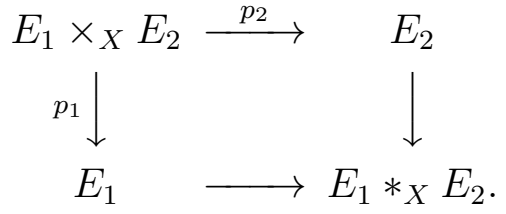

The projections $p_{1}$ and $p_{2}$ are spherical fibrations with odd dimensional fibers. If $E_{1}$ and $E_{2}$ are finitely dominated then the fiber product $E_{1} \times_{X} E_{2}$ and the fiber join $E_{1} *_{X} E_{2}$ are finitely dominated. It follows that one has an equation in $\widetilde{K}_{0}\left(\mathbb{Z} \pi_{1}(X)\right)$ :

$$
\mathcal{O}_{E_{1}}+\mathcal{O}_{E_{2}}=\mathcal{O}_{E_{1} *_{X} E_{2}}+\mathcal{O}_{E_{1} \times_{X} E_{2}}
$$

Since $E_{1} \times_{X} E_{2} \rightarrow E_{2}$ is a spherical fibration with a finitely dominated base and an odd dimensional sphere as the fiber, we conclude that $\mathcal{O}_{E_{1} \times_{X} E_{2}}=0$ (see [20]). Repeated application allows us to prove the lemma.

Lemma 2.8. Let $E \rightarrow X$ be a spherical fibration, where $X$ is a $C W$-complex with k-skeleton $s k_{k} X$. For every integer $k \geq 0$ there is an integer $q$ such that the fibration $E_{k} \rightarrow s k_{k} X$ in the 
cartesian square

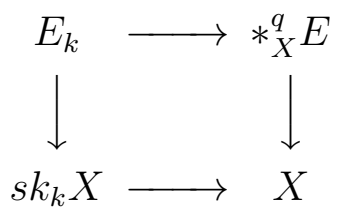

is a product fibration.

Proof. First we observe that by taking fiber joins if necessary (i.e. $E *_{X} E \rightarrow X$ ), we can assume that $E \rightarrow X$ is an orientable spherical fibration.

Let $F$ be a $\mathrm{CW}$-complex. Recall that Aut $F$ denotes the topological monoid of self homotopy equivalences of $F$ and that $\operatorname{Aut}_{I} F$ is the connected component of the identity in Aut $F$. By [4], $B$ Aut $_{I} F$ classifies fibrations $F \rightarrow E \rightarrow B$ for which the map $\pi_{1}(B) \rightarrow[F, F]$ is trivial. In particular, $B$ Aut $_{I} \mathbb{S}^{m}$ classifies orientable spherical fibrations.

The lemma will be proved by induction on $k$; the case $k=0$ being trivial. Now, assume a trivialization over $s k_{k-1} X$; the obstructions to extending this to a trivialization over $s k_{k} X$ are classes $\mathcal{O}_{\Delta} \in \pi_{k-1}\left(\operatorname{Aut}_{I}(F)\right)$, where $F \simeq \mathbb{S}^{m}$ is the fiber and $\Delta$ ranges over the $k$-cells in $X$. Taking the $q$-fold fiber join replaces this obstruction with $q \Sigma_{*}\left(\mathcal{O}_{\Delta}\right)$, where

$$
\Sigma: \operatorname{Aut}_{I}(F) \rightarrow \operatorname{Aut}_{I}\left(*^{q} F\right)
$$

is the natural stabilization map defined by $f \mapsto f * 1 * \cdots * 1$.

Now we know that for a fixed $r>0, \pi_{r}\left(A u t_{I}\left(S^{m}\right)\right) \cong \pi_{r}^{s t}\left(S^{0}\right)$ provided $m$ is sufficiently large. Note that these stable homotopy groups of spheres are all finite. Hence given $k$ as above, we can choose a large integer $q>0$ such that $q \pi_{k-1}\left(A u t_{I}\left(*^{q} F\right)\right)=0$, ensuring the vanishing of all the obstructions and from there the extension of the trivialization.

Theorem 2.9. The cohomology of a connected $C W$-complex $X$ is periodic if and only if there is a spherical fibration $E \rightarrow X$ with a total space $E$ that is homotopy equivalent to a finite dimensional $C W$-complex.

Proof. By taking fiber joins if necessary, we can assume that the spherical fibration is orientable. Now if $E \rightarrow X$ is an oriented spherical fibration and the total space is homotopy equivalent to a finite dimensional complex then the Gysin sequence with local coefficients shows that $X$ has periodic cohomology.

Conversely, assume that $X$ has periodic cohomology. Let $\alpha \in H^{*}(X)$ be an integral cohomology class and $d \geq 0$ be an integer such that $\alpha \cup-: H^{m}(X) \rightarrow H^{m+|\alpha|}(X)$ is an isomorphism for $m \geq d$. By Lemma 2.5, there is an integer $q \geq 1$ and a fibration sequence

$$
P_{d+q|\alpha|-1} \mathbb{S}^{q|\alpha|-1} \rightarrow E \rightarrow X
$$

such that a generator of $H^{q|\alpha|-1}\left(P_{d+q|\alpha|-1} \mathbb{S}^{q|\alpha|-1}\right)$ transgresses to $\alpha^{q} \in H^{q|\alpha|}(X)$. By Lemma 2.6, since $d \geq d-2$ there is an orientable spherical fibration with Euler class $\alpha^{q}$, and the total space has the homotopy type of a finite dimensional complex. 
Let $\Gamma$ denote a discrete group and let $X$ denote a $C W$-complex which is an Eilenberg-MacLane space of type $K(\Gamma, 1)$. We shall say that the group $\Gamma$ has periodic cohomology if $X$ has periodic cohomology.

Corollary 2.10. A discrete group $\Gamma$ has periodic cohomology if and only if $\Gamma$ acts freely and properly on a finite dimensional complex homotopy equivalent to a sphere.

If a countable discrete group $\Gamma$ acts freely and properly on a finite dimensional complex $Y$ homotopy equivalent to a sphere (i.e. $Y$ is a free $\Gamma-\mathrm{CW}$ complex), then it acts freely and properly on $\mathbb{R}^{n} \times \mathbb{S}^{m}$ for some $m, n>0$. Indeed $Y / \Gamma$ has countable homotopy groups, hence is homotopic to a countable complex which in turn is homotopic to an open submanifold $V$ in some Euclidean space; applying the $\mathrm{h}$-cobordism theorem we can infer that for sufficiently large $q$ we have a diffeomorphism $\widetilde{V} \times \mathbb{R}^{q} \cong \mathbb{R}^{n} \times \mathbb{S}^{m}$ for some $m, n>0$ (this appears in [22], lemma 5.4; see also [12], page 139). Conversely the existence of a free and proper $\Gamma$-action on $\mathbb{R}^{n} \times \mathbb{S}^{m}$ implies that $\Gamma$ is countable (as $\mathbb{R}^{n} \times \mathbb{S}^{m}$ is a separable metric space) and that $\Gamma$ has periodic cohomology (via the Gysin sequence). Hence we obtain

Corollary 2.11. A discrete group $\Gamma$ acts freely and properly on $\mathbb{R}^{n} \times \mathbb{S}^{m}$ for some $m, n>0$ if and only if $\Gamma$ is countable and has periodic cohomology.

Remark 2.12. This result represents a proof of a generalized version of a conjecture due to Wall [33] for groups of finite virtual cohomological dimension (that case was verified in [12]). More recently this has been extended to a larger class of discrete groups (see [22]). It is worth noting that the authors in [22] use an abstract notion of periodicity, namely the natural equivalence of functors between $H^{i}(\Gamma$,$) and H^{i+n}(\Gamma$,$) for all i \geq d$. Moreover they speculate that this form of periodicity should be equivalent to the existence of a free and proper action on a finite dimensional homotopy sphere. In view of the results presented here, this question is equivalent to showing that abstract periodicity is always induced by cup product with a cohomology class. This is an interesting open problem in group cohomology.

By a result of Wall [32], a finitely dominated CW-complex $X$ is homotopy equivalent to a finite CW-complex if and only if its finiteness obstruction vanishes; the finiteness obstruction is an element in the reduced projective class group $\widetilde{K}_{0}\left(\mathbb{Z} \pi_{1}(X)\right)$.

Theorem 2.13. Let $X$ be a $C W$-complex of finite type such that the reduced projective class group $\widetilde{K}_{0}\left(\mathbb{Z} \pi_{1}(X)\right)$ is a torsion group. The cohomology of $X$ is periodic if and only if there is a spherical fibration $E \rightarrow X$ such that the total space $E$ is homotopy equivalent to a finite complex.

Proof. As before we can assume orientability for our fibration by taking fiber joins if necessary. Now if $E \rightarrow X$ is an orientable spherical fibration and $E$ is homotopy equivalent to a finite complex then the Gysin sequence shows that $X$ has periodic cohomology.

Conversely, suppose $X$ has periodic cohomology. By Theorem 2.9 there is an orientable spherical fibration $E \rightarrow X$ such that $E$ is homotopy equivalent to a finite dimensional $\mathrm{CW}-$ complex. By forming the fiber join of the map $E \rightarrow X$ with itself if necessary we may assume 
that the fiber of the map $E \rightarrow X$ is a homotopy sphere of odd dimension $2 n-1 \geq 3$ and hence that $\pi_{1}(E)=\pi_{1}(X)$. Since $X$ has finite type, $E$ has finite type. Hence $E$ is finitely dominated and its finiteness obstruction is an element $\mathcal{O}_{E} \in \widetilde{K}_{0}\left(\mathbb{Z} \pi_{1}(X)\right)$. By hypothesis $\mathcal{O}_{E}$ has finite order $q$. The total space of the $q$-fold fiber join $*_{X}^{q} E \rightarrow X$ is also finitely dominated. By Lemma 2.7, the finiteness obstruction of $*_{X}^{q} E$ is $q \mathcal{O}_{E}=0$. Hence $*_{X}^{q} E$ is homotopy equivalent to a finite complex.

Corollary 2.14. Let $\Gamma$ denote a discrete group such that there exists a $C W$-complex of finite type which is an Eilenberg-MacLane space of type $K(\Gamma, 1)$ and such that $\widetilde{K}_{0}(\mathbb{Z} \Gamma)$ is torsion. Then $\Gamma$ has periodic cohomology if and only if it acts freely and properly on a $C W$-complex $Y$ homotopy equivalent to a sphere such that $Y / \Gamma$ is a finite complex.

Remark 2.15. If $G$ is a finite group, $\widetilde{K}_{0}(\mathbb{Z} G)$ is a finite abelian group and of course we can find a $K(G, 1)$ which is a $C W$-complex of finite type.

\section{Group Actions with Periodic Isotropy}

Let $G$ denote a finite group, we will be interested in actions of $G$ on spaces such that the isotropy subgroups have periodic cohomology. The key fact is given by

Proposition 3.1. Let $X$ denote a finite dimensional $G-C W$ complex, where $G$ is a finite group. Then the cohomology of $X \times_{G} E G$ is periodic if and only if all the isotropy subgroups have periodic cohomology.

Proof. Assume that all the isotropy subgroups have periodic cohomology; then we can choose $\alpha \in H^{*}(G, \mathbb{Z})$ such that for any isotropy subgroup $H \subset G, \operatorname{res}_{H}^{G}(\alpha)$ is a periodicity generator (see [1], [5]). If $\alpha$ is described by the extension

$$
0 \rightarrow L_{\alpha} \rightarrow \Omega^{r}(\mathbb{Z}) \rightarrow \mathbb{Z} \rightarrow 0
$$

where $\Omega^{r}(\mathbb{Z})$ denotes the $r$-fold dimension shift of the trivial $\mathbb{Z} G$-module $\mathbb{Z}$, then this is equivalent to the projectivity of the modules $L_{\alpha} \otimes \mathbb{Z}[G / H]$ (see [7], Chapter 5).

Now let $C^{*}(X)$ denote the cellular co-chains on $X$, and consider the exact sequence

$$
0 \rightarrow C^{*}(X) \otimes L_{\alpha} \rightarrow C^{*}(X) \otimes \Omega^{r}(\mathbb{Z}) \rightarrow C^{*}(X) \rightarrow 0 .
$$

By construction, $C^{*}(X) \otimes L_{\alpha}$ is $\mathbb{Z} G$-projective, hence cohomologically trivial-this yields a cohomology isomorphism with any coefficients $M$ for all $i>\operatorname{dim} X$

$$
H^{i}\left(X \times_{G} E G, M\right) \stackrel{\cong}{\longrightarrow} H^{i+|\alpha|}\left(X \times_{G} E G, M\right)
$$

which by construction is induced by multiplying with $\pi^{*}(\alpha)$, where $\pi: X \times_{G} E G \rightarrow B G$ is the usual bundle map.

For the converse observe that periodicity implies that for any prime $p, H^{*}\left(X \times_{G} E G, \mathbb{F}_{p}\right)$ will have Krull dimension one or zero, hence by a theorem due to Quillen [25], all the isotropy must be of rank one i.e. with periodic cohomology. 
The preceding proposition allows us to describe important specific situations where the results from the previous section will apply. In particular, the following theorem is a direct consequence of Theorem 2.9 and Lemma 2.8:

Theorem 3.2. Let $X$ denote a finite dimensional $G-C W$ complex ( $G$ a finite group) such that all of its isotropy subgroups have periodic cohomology. Then there exists a finite dimensional $C W$ complex $Y$ with a free $G$-action such that $Y \simeq \mathbb{S}^{N} \times X$. If $X$ is simply connected and finitely dominated, then we can assume that $Y$ is a finite complex.

Example 3.3. Let $M_{g}$ denote a Riemann surface (orientable) of genus $g>1$. It is well known that $G=\operatorname{Aut}\left(M_{g}\right)$ is a finite group (of order at most $84(g-1)$ ) which acts preserving orientation on $M_{g}$ with isolated singular points (see [16]). Furthermore it is known that the isotropy subgroups of the action are cyclic. Hence we obtain that $G=$ Aut $\left(M_{g}\right)$ acts freely on a finite dimensional complex $Y \simeq \mathbb{S}^{2 n-1} \times M_{g}$.

Remark 3.4. A more direct proof of Theorem 3.2 can be given as follows. Using induction on the dimension of the cells, one can construct a spherical fibration over the skeleta of $X$ one stage at a time. The key ingredients are the existence and homotopy uniqueness of the spherical space forms for the isotropy subgroups and the coherence from the choice of a global periodicity generator; in fact the class $\pi^{*}(\alpha)$ constructed in Proposition 3.1 can be used. The rest of the proof consists of killing obstructions (all of finite exponent) by using fiber joins to extend through higher skeleta, and taking further fiber joins to split the fibration (non-equivariantly). This approach is based on the method that was outlined in [12].

Definition 3.5. The $p$-rank $r_{p}(G)$ of a finite group $G$ is the maximal rank of an elementary abelian $p$-subgroup of $G$, and the rank $r(G)$ of $G$ is $\max _{p|| G \mid} r_{p}(G)$.

Let $G$ be a finite group of rank two. One can choose integral cohomology classes $\alpha, \beta$ in $H^{*}(B G)$ which form a homogeneous system of parameters (see [6]). This can be interpreted in turn as saying that the module $L_{\alpha} \otimes L_{\beta}$ is a projective $\mathbb{Z} G$-module. Under certain conditions this can be used to construct interesting group actions.

Theorem 3.6. Let $G$ be a finite group of rank two. If $\alpha, \beta \in H^{*}(B G, \mathbb{Z})$ are integral cohomology classes which form a homogeneous system of parameters where $\beta$ (of even degree larger than 2) is the Euler class of an oriented spherical fibration $X \rightarrow B G$, then there is a free action of $G$ on a finite $C W$-complex $P$ such that $P$ is homotopy equivalent to a product $\mathbb{S}^{n} \times \mathbb{S}^{m}$ and in the Serre spectral sequence of the fibration $P \times_{G} E G \rightarrow B G$ the generator of $H^{n}\left(\mathbb{S}^{n}, \mathbb{Z}\right)$ transgresses to $\beta$ and the generator of $H^{m}\left(\mathbb{S}^{m}, \mathbb{Z}\right)$ transgresses to a cup power of $\alpha$.

Proof. Let $X \rightarrow B G$ be an oriented spherical fibration having $\beta$ as its Euler class. Since $G$ is finite, $B G$ and hence $X$ are of finite type. Moreover, since $L_{\alpha} \otimes L_{\beta}$ is a projective $G$-module we infer from the Gysin sequence that the map $\alpha \cup-: H^{s}(X, M) \rightarrow H^{s+|\alpha|}(X, M)$ is an isomorphism for every coefficient $M$ and all integers $s$ which are larger than a fixed integer $d$. By Theorem 2.13 there is a spherical fibration $E \rightarrow X$ such that $E$ is homotopy equivalent to a finite $C W$-complex 
and the fundamental class of the fiber transgresses to a cup power of $\alpha$. The $G$ cover of $X$ is homotopy equivalent to a sphere $\mathbb{S}^{n}$ and so the map $\widetilde{X} \rightarrow X$ factors up to homotopy through the $n$-skeleton of $X$. By Lemma 2.8 there is an integer $q$ such that the spherical fibration $*_{X}^{q} E \rightarrow X$ is a product fibration when restricted to the $n$-skeleton of $X$. Hence the map $P \rightarrow \widetilde{X}$ in the cartesian square

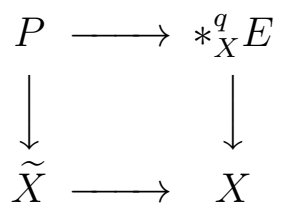

is a product fibration and $P \rightarrow \widetilde{X}$ is a $G$-equivariant spherical fibration such that the fundamental class of the fiber transgresses to a cup power of $\alpha$. So the complex $P$ has the required properties.

From the above we infer that to construct a free action of a rank two finite group on a homotopy product of spheres all we need is an Euler class $\beta$ which forms part of a homogeneous system of parameters. We deal with this question in the next section.

\section{Euler Classes from Actions on Spheres}

In this section we will investigate the existence of group actions on homotopy spheres which have Euler classes representing chosen cohomology classes. Here we assume that all groups which appear are finite. Let $\beta \in H^{N}(G)$ denote a cohomology class which happens to be the Euler class of a $G$-action on $X$, a CW complex homotopic to the $(N-1)$-sphere. In this case the algebraic and topological Gysin sequences provide an identification

$$
H^{i}\left(X \times_{G} E G, \mathbb{Z}\right) \cong H^{i+1}\left(G, L_{\beta}\right) \quad \forall i \geq N .
$$

We recall (see [7] for background)

Definition 4.1. Let $M$ denote any finitely generated $\mathbb{Z} G$-module which is $\mathbb{Z}$-torsion free (a lattice). The complexity $c x_{G}(M)$ of $M$ is the maximal rate of growth of $H^{*}\left(E, M \otimes \mathbb{F}_{p}\right)$ taken over all $p$-elementary abelian subgroups of $G$ and primes $p$ dividing $|G|$.

If $\beta$ is an Euler class, then the complexity of $L_{\beta}$ is determined by the asymptotic growth rates of the $H^{*}\left(E, L_{\beta} \otimes \mathbb{F}_{p}\right)$, and so we have

Proposition 4.2. If $\beta$ is an Euler class for a $G-C W$ complex $X \simeq \mathbb{S}^{N-1}$, then cx $\left(L_{\beta}\right)$ is precisely the maximum of the Krull dimensions of $H^{*}\left(X \times_{G} E G, \mathbb{F}_{p}\right)$, as $p$ ranges over all prime divisors of $|G|$.

Definition 4.3. A cohomology class $\beta \in H^{N}(G, \mathbb{Z})$ is said to be $p$-effective if $c x_{G}\left(L_{\beta} \otimes \mathbb{F}_{p}\right)<$ $r(G)$. The class $\beta$ is said to be effective if it is $p$-effective for all primes $p$ dividing $|G|$ or equivalently if $c x_{G}\left(L_{\beta}\right)<r(G)$. 
Remark 4.4. Note that for a class to be effective it suffices to check that it is $p$-effective for primes of maximal rank.

We assume from now on that $\beta$ is an effective Euler class. Using the lemma above, this will have geometric implications if $X$ is finite dimensional, an assumption we shall make for the rest of this section. It implies that the maximal rank elementary abelian subgroups in $G$ cannot act $^{1}$ with stationary points. In fact we have a simple characterization of effective Euler classes:

Lemma 4.5. If a cohomology class $\beta \in H^{N}(G)$ is the Euler class of an action on a finite dimensional $X \simeq \mathbb{S}^{N-1}$, then $\beta$ is effective if and only if every maximal rank elementary abelian subgroup of $G$ acts without stationary points.

Using the localization theorem in [25], it is clear that this condition can be detected homologically, i.e. $\beta$ is effective if and only if $\left.\beta\right|_{E} \neq 0 \forall E \subset G$ elementary abelian of maximal rank.

Example 4.6. Let $E=(\mathbb{Z} / 2)^{n}$ and $V$ the complex reduced regular representation of $E$, which is $\left(2^{n}-1\right)$-dimensional. Then $V^{E}=\{0\}$ and we obtain an Euler class $e$ associated to the action on $S(V) \cong \mathbb{S}^{2\left(2^{n}-1\right)-1}$. In this case $e \in H^{2\left(2^{n}-1\right)}(E)$ is $D^{2}$, the square of the top Dickson class $D=\prod_{0 \neq z \in H^{1}\left(E, \mathbb{F}_{2}\right)} z$. Note that any proper subgroup of $E$ fixes a point in $S(V)$, i.e. $\left.e\right|_{E^{\prime}}=0$ $\forall E^{\prime} \subsetneq E$.

Euler classes arising from representations are of course fundamental and will play a key role in some of our results. Indeed for $p$-groups it turns out that representations can always be used:

Theorem 4.7. Let $G$ denote a finite $p$-group acting on a finite complex $X$ homotopic to a sphere. Then there exists a real representation $V$ of $G$ such that the Euler class associated to $X$ is effective if and only if the Euler class for $S(V)$ is effective.

Proof. We apply the main result in [13], namely given a complex as above, there exists a real representation $V$ of $G$ such that $\operatorname{dim} V^{H}=\operatorname{dim} X^{H}+1$ for all subgroups $H \subset G$. Applying Lemma 4.5 completes the proof.

From our characterization of effective Euler classes, we see that the problem of finding an effective 'linear' Euler class reduces to finding a representation $V$ such that $V^{E}=\{0\}$ for all $E \subset$ $G$ maximal elementary abelian $p$-subgroups. We provide a construction of such representations for certain groups.

Proposition 4.8. Let $G$ denote a finite group with center $Z(G) \cong \mathbb{Z} / n_{1} \times \cdots \times \mathbb{Z} / n_{k}$ and corresponding generators $x_{1}, \ldots, x_{k}$. Then there exist $[G: Z(G)]$-dimensional complex representations $V_{1}, V_{2}, \ldots, V_{k}$ of $G$ such that $V_{j}^{H}=\{0\}$ for any subgroup $H \subset G$ containing $x_{j}$.

\footnotetext{
${ }^{1}$ Of course it is well-known that if $(\mathbb{Z} / p)^{r}$ acts on a space such as $X$, then there is an index $p$ subgroup fixing a point in $X$.
} 
Proof. Let $\chi_{j}: Z(G) \rightarrow \mathbb{C}$ denote the one-dimensional representation of $Z(G)$ defined by $x_{j} \mapsto$ $e^{2 \pi i / n_{j}}$, and $x_{h} \mapsto 1$ for $h \neq j$. Let $V_{j}=\operatorname{Ind}_{Z(G)}^{G}\left(\chi_{j}\right)$. Using the double coset formula and the fact that $x_{j}$ is central, we readily see that $\left.V_{j}\right|_{Z(G)} \cong \bigoplus^{[G: Z(G)]} \chi_{j}$ and $V_{j}^{\left\langle x_{j}\right\rangle}=\{0\}$.

If $X=S\left(V_{1}\right) \times \cdots \times S\left(V_{k}\right)$ with the diagonal $G$-action, then $Z(G)$ acts freely on $X$. In fact it follows from the results in [14] that if the largest central elementary abelian $p$-subgroup of $G$ has rank equal to $r$, then the associated Euler classes $e_{1}, \ldots, e_{r} \in H^{2[G: Z(G)]}\left(G, \mathbb{F}_{p}\right)$ form a regular sequence in cohomology.

We use these representations ${ }^{2}$ to show

Proposition 4.9. Let $G$ denote a finite group such that the $p$-rank of its center $Z(G)$ is equal to one less than the rank of $r(G)$ of $G$. Then $G$ acts on $X=\left(\mathbb{S}^{2[G: Z(G)]-1}\right)^{r(G)-1}$ such that the isotropy subgroups are all of $p$-rank at most equal to one.

Proof. Take $X$ as before, constructed from the representations induced from the center. If any $E \cong \mathbb{Z} / p \times \mathbb{Z} / p \subset G$ fixed a point in $X$, then $E \cap Z(G)=\{1\}$. Therefore the subgroup generated by $E$ and $Z(G)$ would have $p$-rank at least as large as $r(G)+1$, a contradiction.

Recalling that a $p$-group always has non-trivial center, we obtain

Corollary 4.10. If $G$ is a finite p-group of rank equal to two, then $G$ acts on $X=\mathbb{S}^{2[G: Z(G)]-1}$ with rank one isotropy subgroups.

Applying Theorem 3.2 to the $G-\mathrm{CW}$ complex $X$ (see [31]), we obtain

Theorem 4.11. Let $G$ denote a p-group such that $r=\operatorname{rank} G \leq \operatorname{rank} Z(G)+1$. Then $G$ acts freely on a finite complex

$$
Y \simeq \mathbb{S}^{N} \times\left(\mathbb{S}^{2[G: Z(G)]-1}\right)^{r-1}
$$

In particular, combining the well-known fact $[19]$ that $(\mathbb{Z} / p)^{3}$ does not act freely on $\mathbb{S}^{n} \times \mathbb{S}^{m}$ with Corollary 4.10, we obtain a geometric chacterization of rank two $p$-groups extending Swan's result for periodic groups

Theorem 4.12. A finite p-group $G$ acts freely on a finite complex $Y \simeq \mathbb{S}^{n} \times \mathbb{S}^{m}$ if and only if it does not contain a subgroup isomorphic to $\mathbb{Z} / p \times \mathbb{Z} / p \times \mathbb{Z} / p$.

In the next section we will use linear Euler classes to construct actions of simple groups. It is natural to ask if such spheres will suffice for any rank two group of composite order. Unfortunately this is not the case, in fact the symmetric group $\Sigma_{5}$ has no effective linear Euler classes. One could try instead to use non-linear actions as was done in the spherical space form problem $^{3}$, but this approach is not systematic enough to yield complete results.

\footnotetext{
${ }^{2}$ Note that if $G$ is a $p$-group such that every element of order $p$ is central, the above construction yields a free $G$-action on $X \cong\left(\mathbb{S}^{2}[G: Z(G)]-1\right)^{r(G)}$.

${ }^{3}$ For example the non-abelian group of order 21 does not admit a fixed-point free representation; as shown by Petrie in [24] there is a smooth non-linear action on the 5-dimensional sphere with Euler class a periodicity generator.
} 


\section{Examples: Linear Euler Classes and Actions of Simple Groups.}

In this section we will construct actions of a number of rank two groups on a product of two spheres. The initial input is a representation sphere $S(V)$ which has an Euler class which is effective. We will concentrate on groups which are of rank two at the prime $p=2$, in particular on simple groups.

To put these results in context, we recall a few facts about simple groups. First, note that by the Feit-Thompson Theorem ([17], pg.15), we know that non-abelian simple groups are of even order. Less well-known is that fact that any non-abelian simple group must contain $\mathbb{Z} / 2 \times \mathbb{Z} / 2$ as a subgroup (see [17], pg.13) ${ }^{4}$. Furthermore there is an explicit classification of simple groups $G$ such that their rank at $p=2$ is exactly two (see [17], pg.72). Examining this list and using well-known facts about finite groups of Lie type (see [10]) we obtain the following complete list of rank two simple groups i.e. simple groups which do not contain $(\mathbb{Z} / p)^{3}$ for any prime number $p$ :

$$
\begin{gathered}
\operatorname{PSL}_{2}\left(\mathbb{F}_{p}\right), p \text { odd } p \geq 5 ; \operatorname{PSL}_{2}\left(\mathbb{F}_{p^{2}}\right), p \text { odd } ; \operatorname{PSL}_{3}\left(\mathbb{F}_{p}\right), p \text { odd } ; \\
\operatorname{PSU}_{3}\left(\mathbb{F}_{p}\right), p \text { odd } ; \operatorname{PSU}_{3}\left(\mathbb{F}_{4}\right) ; A_{7} \text { and } M_{11} .
\end{gathered}
$$

On the other hand, one can verify ${ }^{5}$ that all the groups listed above contain a copy of $A_{4}$, the alternating group on four letters. This can be used to obtain a very basic result on actions of rank two simple groups:

Theorem 5.1. If $G$ is a simple group of rank equal to two, then it cannot act freely on a finite dimensional complex $X \simeq \mathbb{S}^{n_{1}} \times \mathbb{S}^{n_{2}}$ via a product action.

Proof. Suppose such an action exists. Recall that the $k$-fold join of $\mathbb{S}^{r}$ is $\mathbb{S}^{k(r+1)-1}$. Hence taking joins we can obtain a free action of $A_{4}$ on $X^{\prime}=\mathbb{S}^{\left(n_{1}+1\right)\left(n_{2}+1\right)-1} \times \mathbb{S}^{\left(n_{1}+1\right)\left(n_{2}+1\right)-1}$, which contradicts a result due to Oliver [23].

Hence to construct actions of these groups one must necessarily consider exotic, non-product actions that go beyond products of representations. It is known (see [26]) that no simple group can act freely on a product of spheres arising from representations, but of course Oliver's criterion and its natural generalization to the groups $(\mathbb{Z} / 2)^{n} \times_{T} \mathbb{Z} /\left(2^{n}-1\right)$ (see [29]) seem more effective in a topological setting.

Consider a group $G$ which contains $\mathbb{Z} / 2 \times \mathbb{Z} / 2$ as a subgroup. We are interested in constructing actions of $G$ on spheres such that the Euler class is effective in mod 2 cohomology. The complex reduced regular representation of $\mathbb{Z} / 2 \times \mathbb{Z} / 2$ gives rise to an orientation-preserving action on $\mathbb{S}^{5}$ with effective mod 2 Euler class. To extend this we make use of the following elementary lemma.

Lemma 5.2. Let $\chi$ be a complex character for $G$ which takes the constant value $\chi(2)$ on all involutions. Then $\left.\chi\right|_{E}$ is the character associated to a multiple of the reduced regular representation of $E \cong \mathbb{Z} / 2 \times \mathbb{Z} / 2$, for every $E \subseteq G$ if and only if $\chi(1)=-3 \chi(2)$.

\footnotetext{
${ }^{4}$ This of course explains why simple groups do not arise in studying spherical space forms.

${ }^{5}$ We are grateful to R. Solomon for explaining this to us.
} 
Proof. The irreducible representations of $E \cong \mathbb{Z} / 2 \times \mathbb{Z} / 2$ are described as follows: if $E=\langle a, b\rangle$ let

$$
\begin{gathered}
\chi_{1}(a)=\chi_{1}(b)=1, \quad \chi_{2}(a)=-1, \quad \chi_{2}(b)=1 \\
\chi_{3}(a)=1, \quad \chi_{3}(b)=-1, \quad \chi_{4}(a)=-1, \quad \chi_{4}(b)=-1 .
\end{gathered}
$$

Then $\left.\chi\right|_{E}=m_{1} \chi_{1}+m_{2} \chi_{2}+m_{3} \chi_{3}+m_{4} \chi_{4}$. Evaluating on $a, b$, ab respectively yields

$$
m_{1}-m_{2}+m_{3}-m_{4}=m_{1}+m_{2}-m_{3}-m_{4}=m_{1}-m_{2}-m_{3}+m_{4}=\chi(2)
$$

whence we readily infer that $m_{2}=m_{3}=m_{4}$ and $m_{1}=\chi(2)+m_{2}$. Hence we deduce that $m_{1}=0$ if and only if $m_{2}=-\chi(2)$; but noting that $\chi(1)=m_{1}+3 m_{2}$ we conclude that $m_{1}=0$ if and only if $\chi(1)=-3 \chi(2)$.

Inspecting the character tables in the Atlas [11], we see that the groups $\Sigma_{4}, A_{5}$ and $\mathrm{SL}_{3}\left(\mathbb{F}_{2}\right)$ have 3-dimensional complex characters which satisfy the conditions of the lemma above.

Theorem 5.3. $\Sigma_{4}, A_{5}$ and $S L_{3}\left(\mathbb{F}_{2}\right)$ act freely on finite complexes $Y \simeq \mathbb{S}^{N} \times \mathbb{S}^{5}$.

Proof. Note that $\left|A_{5}\right|=2^{2} \times 5 \times 3,\left|\Sigma_{4}\right|=2^{3} \times 3,\left|\mathrm{SL}_{3}\left(\mathbb{F}_{2}\right)\right|=2^{3} \times 3 \times 7$, hence for odd primes $p$ they have cyclic $p$-Sylow subgroup and we need only consider the prime $p=2$. By our lemma and the existence of the characters mentioned above, the free actions on $Y \simeq \mathbb{S}^{N} \times \mathbb{S}^{5}$ can be constructed using Theorem 3.2.

Next we consider slightly more complicated simple groups.

Theorem 5.4. The simple group $U_{3}(3)$ acts freely on a finite complex $Y_{1} \simeq \mathbb{S}^{N_{1}} \times \mathbb{S}^{11}$; whereas the simple group $U_{3}(4)$ acts freely on $Y_{2} \simeq \mathbb{S}^{N_{2}} \times \mathbb{S}^{23}$.

Proof. We first consider $G=U_{3}(3)$, note that it has order $2^{5} \cdot 3^{3} \cdot 7$ and that $\operatorname{rank}_{2}(G)=$ $\operatorname{rank}_{3}(G)=2$. Now $P=\operatorname{Syl}_{3}(P)$ is an extra-special 3-group

$$
1 \rightarrow \mathbb{Z} / 3 \rightarrow P \rightarrow \mathbb{Z} / 3 \times \mathbb{Z} / 3 \rightarrow 1
$$

with $\mathbb{Z} / 3 \subset P$ central. Denote by $3 A$ the conjugacy class of this central element of order 3 .

From the Atlas [11] we see that $G$ has one conjugacy class of involutions $(2 A)$ and two conjugacy classes of elements of order three $((3 A)$ and $(3 B)$ respectively). There is a complex character $\chi_{2}$ which takes the following values:

\begin{tabular}{|l|l|l|l|l|}
\hline & 1 & $2 \mathrm{~A}$ & $3 \mathrm{~A}$ & $3 \mathrm{~B}$ \\
\hline$\chi_{2}$ & 6 & -2 & -3 & 0 \\
\hline
\end{tabular}

From these values and the previous lemma, we infer that the associated mod 2 Euler class is effective. However notice that the subgroup generated by $3 \mathrm{~A}$ has no fixed-points, hence acts freely on the associated sphere $S\left(\chi_{2}\right) \cong \mathbb{S}^{11}$. Therefore in mod 3 cohomology the Euler class 
restricts non-trivially to the cohomology of $3 \mathrm{~A}$ and so must be effective. We deduce that $G$ acts on $\mathbb{S}^{11}$ with rank one isotropy and so by our theorem $G$ acts freely on a finite complex $Y_{1} \simeq \mathbb{S}^{N_{1}} \times \mathbb{S}^{11}$

Now we consider $G=U_{3}(4)$; in this case $|G|=2^{6} \cdot 3 \cdot 5^{2} \cdot 13$. According to the Atlas [11], $G$ has only one conjugacy class of involutions, $2 \mathrm{~A}$, and furthermore there is a complex character $\chi$ with the following values

\begin{tabular}{|l|l|l|l|}
\hline & 1 & $2 \mathrm{~A}$ & $5 \mathrm{~A}$ \\
\hline$\chi$ & 12 & -4 & -3 \\
\hline
\end{tabular}

This implies that the mod 2 Euler class for $S(\chi) \cong \mathbb{S}^{23}$ is effective, and as before (but now for $p=5$ ) we also see that $5 \mathrm{~A}$ acts without fixed-points, hence the Euler class is mod 5 effective too. Hence $G$ acts on $\mathbb{S}^{23}$ with periodic isotropy and the rest follows as before.

Example 5.5. $G=\mathrm{GL}_{2}\left(\mathbb{F}_{3}\right),|G|=2^{4} \cdot 3$. We consider a portion of its character table, with a complete set of conjugacy classes:

\begin{tabular}{|l|l|l|l|l|l|}
\hline & 1 & $2 \mathrm{~A}$ & 4 & $2 \mathrm{~B}$ & 3 \\
\hline$\chi_{2}$ & 1 & 1 & 1 & -1 & 1 \\
\hline$\chi_{6}$ & 2 & -2 & 0 & 0 & -1 \\
\hline$\chi_{8}$ & 4 & -4 & 0 & 0 & 1 \\
\hline
\end{tabular}

It is not hard to see that $\mathrm{GL}_{2}(3)$ acts freely on $S\left(\chi_{6}\right) \times S\left(\chi_{2}\right) \cong \mathbb{S}^{3} \times \mathbb{S}^{1}$. From [2] we can think of $H^{*}\left(\mathrm{GL}_{2}(3), \mathbb{F}_{2}\right)$ as the subalgebra of $\mathbb{F}_{2}\left[x_{1}, y_{1}\right]$ generated by $d_{1}=x_{1}+y_{1}, d_{4}=\left(x_{1} y_{1}\right)^{2}$, $d_{3}=x_{1}^{2} y_{1}+x_{1} y_{1}^{2}$, namely

$$
\mathbb{F}_{2}\left[d_{1}, d_{4}, d_{3}\right] / d_{3}^{2}=d_{4} d_{1}^{2} .
$$

$S\left(\chi_{6}\right)$ has Euler class equal to $d_{4}$, whereas $S\left(\chi_{2}\right)$ has Euler class equal to $d_{1}^{2}$.

We can, however, construct an exotic action as follows. Let

$$
\chi=2 \chi_{2}+\chi_{8}
$$

then $\chi(2 A)=\chi(2 B)=-2, \chi(1)=6$. Applying our lemma, we obtain an action of $\mathrm{GL}_{2}(3)$ on $\mathbb{S}^{11}$ with periodic isotropy and hence a free action on $Y=\mathbb{S}^{N} \times \mathbb{S}^{11}$. In this case the class associated to the $\mathbb{S}^{11}$ is $d_{3}^{4}$, and as universal periodicity class $\alpha$ we can take $d_{1}^{4}+d_{4}$, hence $N=4 \ell-1$ and we get $\alpha^{\ell}=\left(d_{1}^{4}+d_{4}\right)^{\ell}$. This exotic action will be used in the sequel. 


\section{Local Euler Classes and Local Subgroups}

As we have seen, linear spheres will often have effective Euler classes. However, there are many groups where this is not the case, the first interesting example is $\Sigma_{5}$, the symmetric group on five letters. Similarly most of the rank 2 simple groups previously listed must be handled in a different way.

To deal with this we introduce local Euler classes and a notion of local equivalence between groups which is flexible enough to substantially extend our collection of examples, at least locally. In our next section we show that effective local Euler classes can be assembled to provide integral effective Euler classes.

Let $\mathbb{S}_{(p)}^{m}$ denote the $p$-local $m$-sphere; so $\mathbb{S}_{(p)}^{1}$ is the classifying space $B \mathbb{Z}_{(p)}$ of the $p$-local integers and for $m>1, \mathbb{S}_{(p)}^{m}$ is a simply connected space with $H_{m}\left(\mathbb{S}_{(p)}^{m}, \mathbb{Z}\right)=\mathbb{Z}_{(p)}$.

Definition 6.1. Let $X$ be a connected CW-complex and let $k \geq 0$ be an integer. A cohomology class $\alpha \in H^{n}\left(X, \mathbb{Z}_{(p)}\right)$ is a $p$-local Euler class if there is a fibration sequence $\mathbb{S}_{(p)}^{n-1} \rightarrow E \rightarrow X$ such that the action of $\pi_{1}(X)$ on $H^{n-1}\left(\mathbb{S}_{(p)}^{n-1}, \mathbb{Z}_{(p)}\right)=\mathbb{Z}_{(p)}$ is trivial and a generator of $H^{n-1}\left(\mathbb{S}_{(p)}^{n-1}, \mathbb{Z}_{(p)}\right)$ transgresses to $\alpha$.

Definition 6.2. Let $G$ and $H$ denote discrete groups. We say that they are $p$-equivalent (denoted $G \simeq_{p} H$ ) for a prime $p$ if the localizations at $p$ of their classifying spaces are homotopy equivalent, $B G_{(p)} \simeq B H_{(p)}$.

Note that if we are given two $p$-equivalent groups such that one of them is finite, then the notion of $p$-effective Euler class makes sense for both of them.

Definition 6.3. A $p$-effective Euler class for a discrete group $G$ is a cohomology element $x \in$ $H^{2 n}\left(B G, \mathbb{Z}_{(p)}\right)$ which is of the form $f^{*}(y)$, where $f: B G_{(p)} \rightarrow B H_{(p)}$ is an $H \mathbb{Z}_{(p)}$-equivalence and $y \in H^{2 n}\left(B H_{(p)}, \mathbb{Z}_{(p)}\right)$ is a $p$-effective Euler class for the finite group $H$.

We will be interested in constructing local actions, i.e. actions on the localization $\mathbb{S}_{(p)}^{N-1}$ with effective mod $p$ Euler classes. A basic result is explained in the following lemma, which will be proved in the following section.

Lemma 6.4. Assume that we have two discrete groups $G$ and $H$ which are $p$-equivalent, where one of them is finite. Then there exists an action of $G$ on $\mathbb{S}_{(p)}^{2 k-1}$ (a p-local sphere) with a $p$-effective Euler class if and only if the same holds for $H$.

Example 6.5. Using a double coset decomposition one can see that the natural inclusion $\Sigma_{p^{n}} \subset$ $\Sigma_{p^{n}+1}\left(p\right.$ a prime) induces an isomorphism in mod $p$ cohomology. Hence $\Sigma_{5}$ will act on a 2 -local sphere with effective Euler class (as this holds for $\Sigma_{4}$ ).

Example 6.6. If $G$ is a finite group with abelian $p$-Sylow subgroup $A$, then (by a theorem of Swan), the inclusion of the normalizer $N_{G}(A)$ in $G$ induces a mod $p$ cohomology equivalence. (see [2]). 
Proposition 6.7. If a finite group $G$ has a normal $p$-Sylow subgroup, then its cohomology has a $p$-effective Euler class. Hence if $G$ has an abelian p-Sylow subgroup it has a p-effective Euler class.

Proof. Let $G$ denote a group with normal $p$-Sylow subgroup $P \triangleleft G$. Consider an effective class $\alpha$ for $P$ arising from a $[P: Z(P)]$-dimensional representation of $P$ as in Proposition 4.8. Now induce this to a representation for $G$; by the double coset formula, the resulting Euler class will restrict in $H^{*}\left(P, \mathbb{F}_{p}\right)$ to $\prod_{g \in G / P} g \alpha$, which is evidently also an effective Euler class.

Next we describe a method for producing characters for discrete groups which arise as the fundamental group $\Gamma$ of a graph of finite groups (see [9], p. 179 and [28]). Recall that associated to such a group we have a finite connected graph $Y$ together with groups $G_{v}$ and $G_{e}$ where $v$ (respectively $e$ ) ranges over the vertices (respectively edges) of $Y$. We also have monomorphisms $\theta_{0}: G_{e} \rightarrow G_{v}, \theta_{1}: G_{e} \rightarrow G_{w}$ for every edge $e$ with vertices $v$ and $w$. There is a tree $T$ with an action of $\Gamma$ such that the orbit space of the action is precisely $Y$. The group $\Gamma$ can be thought of as a generalized amalgam obtained as a quotient of the free product of the vertex groups $G_{v}$ by the smallest normal subgroup generated by the relations $\theta_{0}(a)=\theta_{1}(a)$ for all $a \in G_{e}$.

Now fix a group $Q$, and suppose we have homomorphisms $f_{v}: G_{v} \rightarrow Q$ such that $f_{v} \theta_{0}=f_{w} \theta_{1}$ for all pairs of vertices inciding on a common edge. This uniquely determines a homomorphism $F: \Gamma \rightarrow Q$. In particular if $Q=G L_{n}(\mathbb{C})$, we can use this approach to construct linear representations for generalized amalgams.

The following theorem makes this useful for constructing Euler classes for a rank two group $G$. Let $\left|A_{p}(G)\right|$ denote the geometric realization of the poset of non-trivial $p$-elementary abelian subgroups in $G$ (see [2] for properties). Note that conjugation induces a natural $G-\mathrm{CW}$ complex structure on this space.

Proposition 6.8. Let $G$ denote a finite group such that $\left|A_{p}(G)\right|$ is a path-connected graph. If $\Gamma$ denotes the fundamental group of the graph of groups associated to $Y=\left|A_{p}(G)\right| / G$, then the natural surjection $p: \Gamma \rightarrow G$ induces a $\mathbb{Z}_{(p)}$-cohomology isomorphism.

This is a special case of a theorem due to Brown (see [9] and also [34]). The key fact is that $H^{1}\left(\left|A_{p}(G)\right|, \mathbb{Z}_{(p)}\right)$ is a projective $\mathbb{Z}_{(p)} G$-module (analogous to the Steinberg module). It is worthwhile to point out that $\pi=\operatorname{ker} p=\pi_{1}\left(\left|A_{p}(G)\right|\right)$ is a free group of finite rank and that $\Gamma$ can be identified with $\pi_{1}\left(\left|A_{p}(G)\right| \times_{G} E G\right)$. In this case the tree $T$ is simply the universal cover $\left|\widetilde{A_{p}(G)}\right|$. Note that $\left|A_{p}(G)\right|$ is path connected if and only if $\Gamma \rightarrow G$ is surjective.

More generally, (as explained in [34], pg. 151-152) $G$ acts transitively on the components of $\left|A_{p}(G)\right|$, with a stabilizer $N$ which is $p$-equivalent to $G$ via the restriction map. Hence for our purposes we can always replace $G$ with this stabilizer group and so apply the previous result to construct Euler classes for a rank two group by using a $p$-equivalent group $\Gamma$. In other words we make use of the equivalences $G \simeq_{p} N \simeq_{p} \Gamma$.

Our strategy will be to glue together characters on local subgroups to yield characters for generalized amalgams, and then use the associated localized spheres to produce effective Euler 
classes. Note that the Euler class associated to a $\Gamma$-sphere $W$ will be effective if and only if it restricts to an effective Euler class on each of the generating finite subgroups. Hence we need to assemble characters for the generalized amalgams which arise from characters with effective $\mathbb{Z}_{(p)}$-Euler classes.

Example 6.9. $G=M_{11}, p=2$. In this case (see [2]) we can express $M_{11}$ as a quotient of $\Gamma=\mathrm{GL}_{2}(3) *_{D_{8}} \Sigma_{4}$ with $\Gamma \rightarrow G$ inducing a mod 2 equivalence. Consider $\chi=2 \chi_{2}+\chi_{8}$ the representation for $\mathrm{GL}_{2}(3)$ which we used previously, and compare it with the following character for $\Sigma_{4}$ (also used previously):

\begin{tabular}{|l|l|l|l|l|l|}
\hline & 1 & $(12)$ & $(12)(34)$ & $(1234)$ & $(123)$ \\
\hline$\nu$ & 3 & -1 & -1 & 1 & 0 \\
\hline
\end{tabular}

Then clearly $\chi$ agrees with $2 \nu$ on elements of order 2 and 4 , hence on the subgroup $D_{8}$. Therefore we have a representation $V$ (6-dimensional) for $\Gamma$ which affords an effective Euler class mod 2 (indeed it restricts to effective classes on the two conjugacy classes of maximal finite subgroups). We obtain a 2 -effective Euler class in $H^{12}\left(M_{11}, \mathbb{F}_{2}\right)$. Note that $V=\mathbb{Z} / 3 \times \mathbb{Z} / 3=S y l_{3}\left(M_{11}\right)$, with normalizer of order 144 . Hence we may find a 3 -local sphere of dimension 31 providing a 3-effective Euler class in $H^{32}\left(M_{11}, \mathbb{F}_{3}\right)$.

Example 6.10. $G=\mathrm{PSL}_{2}\left(\mathbb{F}_{q}\right) q$ odd, $q \equiv \pm 3 \bmod 8$. In this case the 2-Sylow subgroup is elementary abelian and by Proposition 6.7 we can obtain a 2-effective Euler class. For the case $G=\mathrm{PSL}_{2}\left(\mathbb{F}_{q}\right) q$ odd, $q \equiv \pm 1 \bmod 8$, we observe that the group has two conjugacy classes of rank two elementary abelian subgroups (both with normalizers isomorphic to $\Sigma_{4}$ ), and one conjugacy class of involutions, with centralizer a dihedral group $D_{2 r}$ (here $r$ depends on the prime $p$ ). The corresponding intersections are both $D_{8}$, hence we can easily construct a 3-dimensional representation for all of these stabilizers with the desired property. We obtain a 2-effective Euler class in $H^{6}\left(\mathrm{PSL}_{2}\left(\mathbb{F}_{q}\right), \mathbb{F}_{2}\right)$.

Example 6.11. $G=\operatorname{PSL}_{3}\left(\mathbb{F}_{q}\right) q$ odd, $q \equiv-1 \bmod 3$. Here the 2-local structure is

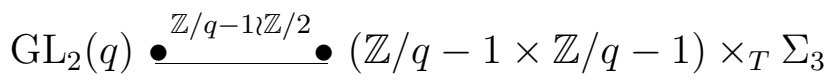

Take $\mathbb{Z} / 2 \subset \mathbb{Z} / q-1 \times \mathbb{Z} / q-1 \subset \mathrm{GL}_{2}(q)$ and choose $\chi$ a 1 -dimensional representation of $\mathbb{Z} / q-1 \times \mathbb{Z} / q-1$ such that $\left.\chi\right|_{\mathbb{Z} / 2}$ is non-trivial. Let $\nu=\operatorname{Ind}_{\mathbb{Z} / q-1 \times \mathbb{Z} / q-1}^{G L_{2}(q)}(\chi)$; then this is a $q(q+1)$ dimensional representation on which $\mathrm{SL}_{2}(q)$ acts 2 -freely. Now taking $\mathrm{GL}_{2}(q) / \mathrm{SL}_{2}(q) \cong \mathbb{Z} / q-1$ and selecting a 1-dimensional embedding $\mathbb{Z} / q-1 \subset \mathbb{S}^{1}$ we obtain a character $\omega$ such that every element of even order in $\mathrm{GL}_{2}(q)$ acts freely on $S(\nu) \times S(\omega)$. Now take

$$
\mathcal{K}=2 \nu+q(q+1) \omega .
$$


This is a $3 q(q+1)$-dimensional representation. Let $2 \mathrm{~A}$ denote the central involution, $2 \mathrm{~B}$ the non-central one, then

$$
\mathcal{K}(2 A)=-q(q+1), \mathcal{K}(2 B)=-q(q+1)
$$

and hence $S(\mathcal{K})$ has an effective Euler class $\bmod 2$.

Next we consider $\mathrm{SL}_{3}\left(\mathbb{F}_{q}\right) \supset(\mathbb{Z} / q-1 \times \mathbb{Z} / q-1) \times_{T} \Sigma_{3}$, where this action arises from $\mathbb{Z} / q-12 \Sigma_{3} \subset$ $\mathrm{GL}_{3}\left(\mathbb{F}_{q}\right)$ using the fact that diagonal matrices of determinant one are permuted by $\Sigma_{3}$.

Now let $\mathbb{Z} / q-1 \stackrel{\rho}{\hookrightarrow} U(1)$ be the standard 1-dimensional representation given by taking a generator to a primitive $(q-1)$-root of unity; this gives rise to a representation

$$
(\mathbb{Z} / q-1)^{2} \times_{T} \Sigma_{3} \hookrightarrow U(1) \imath \Sigma_{3} \subset U(3)
$$

and so we obtain a 3 -dimensional character $\zeta$ for $(\mathbb{Z} / q-1 \times \mathbb{Z} / q-1) \times_{T} \Sigma_{3}$. By naturality $S(\zeta)$ has an effective 6-dimensional Euler class which restricts to the square of the top Dickson class on all $\mathbb{Z} / 2 \times \mathbb{Z} / 2$ subgroups - indeed one checks that $\left.\zeta\right|_{\mathbb{Z} / 2 \times \mathbb{Z} / 2}$ is a copy of the reduced regular representation. It is direct to verify that $q(q+1) \zeta$ agrees with $\mathcal{K}$ on $\mathbb{Z} / q-l \imath \mathbb{Z} / 2$, and so we can construct a $6 q(q+1)-1$ dimensional sphere associated to the amalgam $\Gamma$ providing an effective $\bmod 2$ cohomology class. Observe that if $q \equiv 1 \bmod 3$, then suitably modified arguments produce a similar result, where we must divide out by the central $\mathbb{Z} / 3$ in the diagram above.

Example 6.12. For the groups $U_{3}(q), q \equiv 1 \bmod 3$, we have the following diagram of subgroups ${ }^{6}$

$$
\mathrm{GU}_{2}(q) \bullet \bullet^{\mathbb{Z} / q+12 \mathbb{Z} / 2} \bullet(\mathbb{Z} / q+1 \times \mathbb{Z} / q+1) \times_{T} \Sigma_{3}
$$

A construction similar to the previous one produces the desired linear sphere for the amalgam. In the case $q \equiv-1 \bmod 3$ we must divide by a central $\mathbb{Z} / 3$ in the diagram above and modify the construction accordingly.

The inclusion $A_{6} \hookrightarrow A_{7}$ induces a mod 2 equivalence, hence given the isomorphism $A_{6} \cong$ $P S L_{2}\left(\mathbb{F}_{9}\right)$ we see that $A_{7}$ has an effective 2-local Euler class. Thus we have completed arguments for all the simple rank 2 groups on our list, and our results can be collected together as follows:

Theorem 6.13. Let $G$ denote a finite simple group of rank equal to two. Then $G$ acts on some $Y \simeq \mathbb{S}_{(2)}^{2 k-1}$ such that the associated Euler class is 2-effective.

Odd primes are in most instances easier to handle than $p=2$. If $q$ is an odd prime, then the $q$-Sylow subgroups of the $P S L_{2}\left(\mathbb{F}_{p}\right)$ and $P S L_{2}\left(\mathbb{F}_{p^{2}}\right)$ are all cyclic (as the corresponding $S L_{2}$ have rank one). In addition the odd order Sylow subgroups for $A_{7}$ and $M_{11}$ are all abelian and we have already seen how to construct locally effective Euler classes in that case. The only complications may arise either at $p=3$ or at the defining characteristic for $U_{3}(p)$ and $L_{3}(p)$. It is however elementary to check that for any rank 2 simple group other than $U_{3}(3)$ or $L_{3}(3)$ the 3-Sylow subgroup is abelian-and of course we have already dealt with $U_{3}(3)$ in the previous section. The following lemma takes care of $U_{3}(p)$ at the characteristic

\footnotetext{
${ }^{6}$ Again we are grateful to R.Solomon for sketching a description of $A_{2}\left(U_{3}(p)\right)$.
} 
Lemma 6.14. Let $G=U_{3}(p)$, the unitary group over $\mathbb{F}_{p}$. Then $G$ has a unique cuspidal unipotent representation and it has degree $p(p-1)$. Furthermore the associated Euler class in $H^{2 p(p-1)}(G, \mathbb{Z})$ is p-effective.

Proof. The first part can be found in [21], page 174. For the second part we use an argument due to G.Seitz ([27]). Note that $p(p-1)$ is the minimal degree of a complex irreducible character for $G$. This is also the minimal degree for a faithful irreducible of the Sylow normalizer, which is a semidirect product $P \times_{T} \mathbb{Z} / p-1$, where $P=\operatorname{Syl}_{p}(G)$ is extra-special of order $p^{3}$. The cyclic group $\mathbb{Z} / p-1$ acts transitively on the non-trivial linear characters of $Z(P)$. The given representation restricts to $\operatorname{Syl}_{p}(G)$ as the sum of the $p-1$ irreducibles of degree $p$. Restricting further to $Z(P)$ we just get all non-trivial linear characters, each $p$ times. This has value $-p$ on each element of order $p$ in the center, in particular any such element acts without fixed-points on the associated linear sphere. We infer from Lemma 4.5 that the corresponding Euler class must be $p$-effective.

To summarize the results in this section, we can state:

Theorem 6.15. Given any rank two simple group $G$ and any prime $q$ dividing $|G|$ (except possibly $\mathrm{PSL}_{3}\left(\mathbb{F}_{p}\right)$ at $\left.p\right)$, then it has a q-effective Euler class in its cohomology.

\section{Hомотору Actions}

In this section we prove Lemma 6.4 and show that effective Euler classes can be obtained by gluing together $p$-effective Euler classes.

Let $F$ be a $\mathrm{CW}$-complex. Then Aut $F$ is the topological monoid of self homotopy equivalences of $F$ and $\operatorname{Aut}_{I} F$ is the component of the identity in Aut $F$. By [4] there is a universal fibration with base the classifying space $B$ Aut $F$ and homotopy fiber $F$ such that, for $X$ a $C W$-complex, the fiber homotopy equivalence classes of fibrations with homotopy fiber $F$ and base $X$ are in natural one to one correspondence with the homotopy classes of maps from $X$ to $B$ Aut $F$. Let $G$ be a topological group, the equivariant homotopy classes of free $G$-CW complexes are in a natural one-to-one correspondence with the fiber homotopy equivalence classes of fibrations with base $B G$. Combining these results, the homotopy equivalence classes of maps $B G \rightarrow B$ Aut $F$ are in one-to-one correspondence with the homotopy actions of $G$ on $F$.

For a set of primes $K$, let $\mathbb{Z}_{K}$ be the smallest subring of the rationals that contains the reciprocals of the prime numbers not in $K$. A simply-connected $\mathrm{CW}$-complex is $\mathbb{Z}_{K}$-local if one of two equivalent conditions hold: its integral homology groups are $\mathbb{Z}_{K}$-modules or its homotopy groups are $\mathbb{Z}_{K}$-modules (the equivalence comes from Serre $\mathcal{C}$-theory). The localization of a simply-connected CW-complex $X$ is a CW-complex $X_{K}$ and a map $X \rightarrow X_{K}$ (called the localization map) such that the $X_{K}$ is $\mathbb{Z}_{K^{-}}$local and the induced map $H_{*}(X, \mathbb{Z}) \otimes \mathbb{Z}_{K} \rightarrow$ $H_{*}\left(X_{K}, \mathbb{Z}\right)$ is an isomorphism.

We recall a standard result in homotopy theory (see [15], 2.1).

Proposition 7.1. Let $K$ denote any set of primes in $\mathbb{Z}$, then $B$ Aut $_{I} \mathbb{S}_{K}^{n}$ is a $\mathbb{Z}_{K}$-local space. 
Using the above we can now prove Lemma 6.4. Suppose given two $p$-equivalent groups $G$ and $H$ (with one of them finite) and an action of $G$ on $\mathbb{S}_{(p)}^{n}$ with $p$-effective Euler class. As $B$ Aut $\mathbb{S}_{(p)}^{n}$ is $p$-local, the corresponding map $B G \rightarrow B$ Aut $_{I} \mathbb{S}_{(p)}^{n}$ factors through $B G_{(p)}$. Now using the $p$ equivalence and the natural localization map we obtain a homotopy class in $\left[B H, B \operatorname{Aut}_{I}\left(\mathbb{S}_{(p)}^{n}\right)\right]$ which by naturality must give rise to an action with $p$-effective Euler class.

Next we make a small digression to outline how local homotopy actions can be assembled to define a global action. Let $J$ and $K$ be sets of prime numbers. Then (see [8]) there is a homotopy pullback square of localization maps

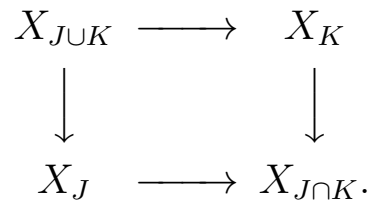

The map $\operatorname{hom}\left(X_{J}, X_{J}\right) \rightarrow \operatorname{hom}\left(X, X_{J}\right)$ induced by the localization map is a weak equivalence and so there is a homotopy pullback square

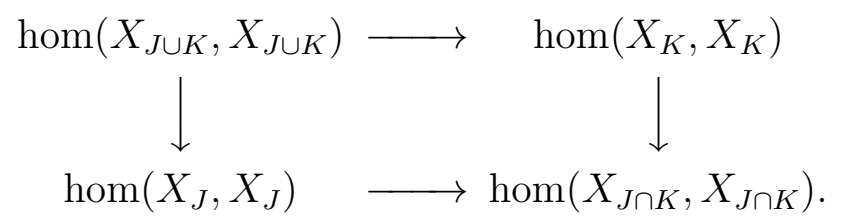

The localization $f_{J \cup K}$ of a map $f$ is a weak equivalence if and only if the maps $f_{J}$ and $f_{K}$ are weak equivalences and so there is a homotopy pullback square of monoids

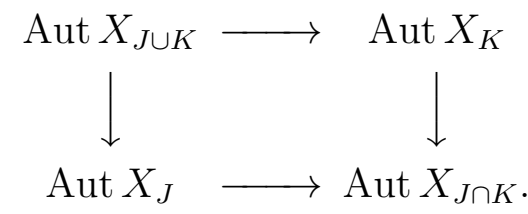

If $X$ is a finite nilpotent complex, the localization $f_{J \cup K}$ of the self map $f: X \rightarrow X$ is homotopic to the identity map on $X_{J \cup K}$ if and only if the self maps $f_{J}$ and $f_{K}$ are homotopy equivalent to the identity maps on $X_{J}$ and $X_{K}$. So, there is a homotopy pullback square of monoids

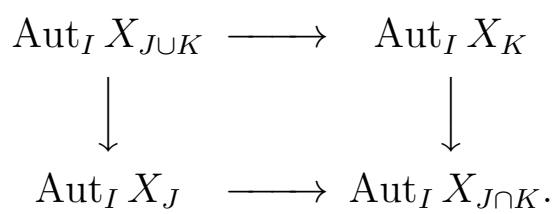

Constructing the classifying spaces of the monoids gives a homotopy pullback square

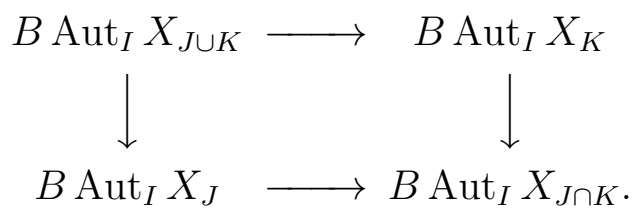

This pull-back construction will be used to assemble together local homotopy actions. 
We are now ready to consider the problem of gluing together $p$-effective Euler classes for a finite group $G$. An integral cohomology class $\alpha \in H^{n}(B G, \mathbb{Z})$ is an Euler class if there is a spherical fibration $\mathbb{S}^{n-1} \rightarrow E \rightarrow B G$ such that the fundamental class in $H^{n-1}\left(\mathbb{S}^{n-1}, \mathbb{Z}\right)$ transgresses to $\alpha$. It is an effective Euler class if its restriction to every elementary abelian subgroup of maximal rank is non-trivial. A $p$-local cohomology class $\alpha \in H^{n}\left(B G, \mathbb{Z}_{(p)}\right)$ is a a $p$-local Euler class if there is a fibration $\mathbb{S}_{(p)}^{n-1} \rightarrow E \rightarrow B G$ such that the fundamental class in $H^{n-1}\left(\mathbb{S}_{(p)}^{n-1}, \mathbb{Z}_{(p)}\right)$ transgresses to $\alpha$. It is an effective $p$-local Euler class if its restriction to every elementary abelian $p$-subgroup of maximal rank is nontrivial. In this section we show that the problem of constructing effective Euler classes can be solved one prime at a time.

Theorem 7.2. An integral cohomology class $\alpha \in H^{2 n}(B G, \mathbb{Z})$ with $n \geq 2$ is an effective Euler class if and only if for each prime $p$ such that $r k G=r k_{p} G$, its p-localization $\alpha_{p} \in H^{2 n}\left(B G, \mathbb{Z}_{(p)}\right)$, is an effective p-local Euler class.

Proof. Clearly an Euler class is effective if and only if its $p$-localization at each prime for which $r k G=r k_{p} G$ is effective. Moreover if $\alpha$ is an Euler class then each of its localizations $\alpha_{p}$ is a $p$-local Euler class.

Now assume that $\alpha \in H^{2 m}(B G, \mathbb{Z})$ and that for each prime $p$ for which $r k G=r k_{p} G$ one has a fibration $E_{p} \rightarrow B G$ with homotopy fiber the $p$-local sphere $\mathbb{S}_{(p)}^{2 m-1}$ and $p$-local Euler class $\alpha_{p}$. So $\pi_{1}\left(E_{p}\right)=G$ and the universal cover of $E_{p}$ is a $G$-CW complex that is homotopy equivalent to $\mathbb{S}_{(p)}^{2 m-1}$. Since the fundamental class survives to $E_{2}$ in the Serre spectral sequence, the action of $G$ on the $p$-local homotopy sphere is homologically trivial and hence homotopically trivial. Let $p_{1}, p_{2}, \ldots, p_{k}$ be the finite set of primes for which $r k G=r k_{p} G$ and let $K$ be the set of all other primes. There is a unique homologically trivial action of $G$ on the rationalization $\mathbb{S}_{0}^{2 m-1}$ of an odd dimensional sphere (here all primes are inverted). Give $\mathbb{S}_{K}^{2 m-1}$ the trivial action of $G$. Then one has a diagram of $G$-CW complexes

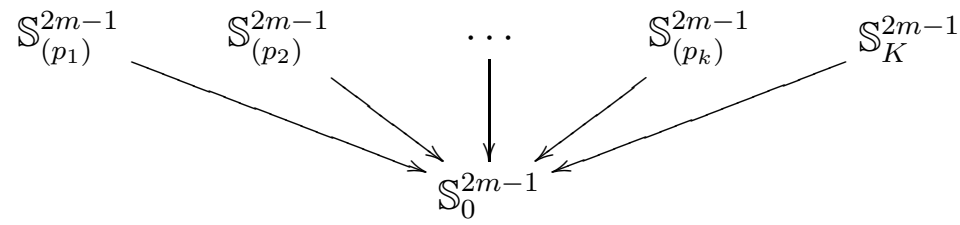

Using the homotopy pullback diagram for the classifying spaces described previously, we see that the homotopy classes defining the local homotopy actions define a homotopy action of $G$ on the homotopy pull-back of the diagram, which by the fracture lemma of Bousfield-Kan (see [8], page 147) is homotopy equivalent to $\mathbb{S}^{2 m-1}$. From this we obtain a $G-\mathrm{CW}$ complex whose associated Borel construction gives the required spherical fibration

$$
\mathbb{S}^{2 m-1} \rightarrow \mathbb{S}^{2 m-1} \times_{G} E G \rightarrow B G .
$$

Our methods yield actions on homotopy spheres which are hard to realize geometrically. In particular they allow us to construct effective Euler classes for groups where linear spheres are 
not useful. In turn we may use these spheres to construct free group actions on finite complexes. Using fiber joins and applying Theorem 6.7 and Theorem 6.15 respectively, we obtain

Theorem 7.3. Let $G$ denote a rank two finite group such that all of its $p$-Sylow subgroups are either normal in $G$, abelian or generalized quaternion. Then $G$ acts freely on a finite complex $Y \simeq \mathbb{S}^{n} \times \mathbb{S}^{m}$

Theorem 7.4. Let $G$ denote a rank two finite simple group other than $P S L_{3}\left(\mathbb{F}_{p}\right)$, $p$ an odd prime. Then $G$ acts freely on a finite complex $Y \simeq \mathbb{S}^{n} \times \mathbb{S}^{m}$.

We conclude with an example illustrating the theorem above.

Example 7.5. Let $G=M_{11}$, the first Mathieu group. As we have seen it has a effective Euler classes for $p=2$ (in dimension 12) and for $p=3$ (in dimension 32), where $p=2,3$ are the only two maximal rank primes. Hence we obtain that $M_{11}$ acts freely on a finite complex $Y \simeq \mathbb{S}^{N} \times \mathbb{S}^{383}$ (note that $\left.*^{32} \mathbb{S}^{11} \simeq *^{12} \mathbb{S}^{31} \simeq \mathbb{S}^{383}\right)$

\section{REFERENCES}

[1] Adem, A., Torsion in Equivariant Cohomology Comm. Math. Helv. 64, pp. 401-411 (1989).

[2] Adem, A. and Milgram, R.J., Cohomology of Finite Groups, Springer-Verlag Grundlehren 309 (1994).

[3] Adem, A. and Smith, J.H., On Spaces with Periodic Cohomology, Electronic Research Announcements of the American Mathematical Society 6 (2000).

[4] Barratt, M., Gugenheim, V. and Moore, J., On semisimplicial fibre-bundles, Amer. J. Math. 81, pp. 639-657 (1959).

[5] Benson, D. and Carlson, J., Complexity and Multiple Complexes, Math. Zeit. 195, pp. 221-238 (1987).

[6] Benson, D. and Carlson, J., Projective Resolutions and Poincaré Duality Complexes, AMS Transactions 342, pp. 447-488 (1994).

[7] Benson, D., Representations and Cohomology II, Cambridge U. Press (1991).

[8] Bousfield, A.K. and Kan, D.M., Homotopy Limits, Completions and Localizations, Springer-Verlag Lecture Notes in Mathematics 304 (1972).

[9] Brown, K., Cohomology of Groups, Springer-Verlag Graduate Texts in Mathematics 87 (1982).

[10] Carter, R., Finite Groups of Lie Type J. Wiley and Sons (1985).

[11] Conway, J. et al. Atlas of Finite Groups, Oxford U. Press (1985).

[12] Connolly, F. and Prassidis, S., Groups Which Act Freely on $R^{m} \times S^{n-1}$, Topology 28 pp. $133-148$ (1989).

[13] Dotzel, R. and Hamrick, G., p-Group Actions on Homology Spheres, Inventiones Math. 62, pp. 437-442 (1981).

[14] Duflot, J., Depth and Equivariant Cohomology, Comm. Math. Helv. 56, pp. 627-637 (1981).

[15] Dwyer, W.G., R-Nilpotency in Homotopy Equivalences, Israel J. Math. 66 pp. 154-159 (1989)

[16] Farkas, H.M. and Kra, I., Riemann Surfaces Springer-Verlag 1980.

[17] Gorenstein, D., The Classification of Finite Simple Groups Plenum Press (1983).

[18] Gray, B., Homotopy Theory, Academic Press 1975.

[19] Heller, A., A Note on Spaces with Operators, Illinois Journal of Mathematics 3, pp. 98-100 (1959).

[20] Lueck, W., The Transfer Maps Induced in the Algebraic $K_{0}$ and $K_{1}$ Groups by a Fibration I, Math. Scand. 59, pp. 93-121 (1986).

[21] Lusztig, G., Irreducible Representations of Finite Classical Groups, Inventiones Mathematicae 43, pp. 125175 (1977). 
[22] Mislin, G. and Talelli, O., On Groups which Act Freely and Properly on Finite Dimensional Homotopy Spheres, Computational and geometric aspects of modern algebra (Edinburgh 1998), pp. 208-228, London Math. Soc. Lecture Note Series 275, Cambridge University Press (2000).

[23] Oliver, R., Free Compact Group Actions on Products of Spheres, Springer-Verlag LNM 763, pp. 539-548 (Arhus 1978).

[24] Petrie, T., Free Metacyclic Group Actions on Homotopy Spheres, Annals of Mathematics 94, pp. 108-124 (1971).

[25] Quillen, D., The Spectrum on an Equivariant Cohomology Ring I, Annals of Mathematics 94, pp. 549-572 (1971).

[26] Ray, U., Free Linear Actions of Finite Groups on Products of Spheres, Journal of Algebra 147, pp. 456-490 (1992).

[27] Seitz, G., Private communication.

[28] Serre, J.-P., Arbres, Amalgams, $S L_{2}$, Asterisque 46 (1977).

[29] Silverman, J., Subgroup Conditions for Groups Acting Freely on Products of Spheres, AMS Transactions 334, pp. 153-181 (1992).

[30] Swan, R.G., Periodic Resolutions for Finite Groups, Annals of Mathematics 72 pp. 267-291 (1960).

[31] tom Dieck, T., Transformation Groups, de Gruyter Studies in Math. 8 (1987).

[32] Wall, C.T.C., Finiteness Conditions for CW-complexes II, Proceedings Royal Society, Series A 295, pp. 129-139 (1966).

[33] Wall, C.T.C., Periodic Projective Resolutions, Proc. London Math. Soc. 39 pp. 509-533 (1979).

[34] Webb, P., A Local Method in Group Cohomology, Comm. Math. Helv. 62, pp. 135-167 (1987).

Department of Mathematics, University of Wisconsin, Madison, Wisconsin 53706

E-mail address: adem@math.wisc.edu

Department of Mathematics, Purdue University, West Lafayette, Indiana 47907

E-mail address: jhs@math.purdue.edu 